introduced in Italy in 1982. To determine the success of this program, Corrado et al. studied 12-35-year-old athletes and nonathletes in the Veneto region of Italy from 1979 to 2004 . Over this period, screened athletes experienced 1.9 SCDs per 100,000 person-years - with nearly all SCDs experienced during or shortly after sports activity-compared with 0.79 SCDs per 100,000 person-years in unscreened nonathletes. While the annual incidence of SCD changed little in the nonathletes during the study period, there was an $89 \%$ decrease among screened athletes $\left(P_{\text {trend }}<0.001\right)$. A marked decrease in the number of sudden deaths from cardiomyopathies was largely responsible for the decline; a parallel sports medical center study of disqualifications from competitive sports showed that the proportion of athletes disqualified for cardiomyopathies doubled from the early to the late screening period.

Data from this one region, therefore, seem to support systematic screening of athletes for cardiomyopathies before sports participation, and indicate that such a program should also be considered in other countries.

Original article Corrado D et al. (2006) Trends in sudden cardiovascular death in young competitive athletes after implementation of a preparticipation screening program. JAMA 296: 1593-1601

\section{Peripheral arterial disease raises mortality risk after percutaneous coronary intervention}

Peripheral arterial disease (PAD) often coexists with other forms of systemic atherosclerosis, and has been linked with poor outcomes and postprocedural complications. Saw et al. have assessed the effects of concomitant PAD on mortality and ischemic events in patients who underwent percutaneous coronary intervention $(\mathrm{PCl})$ for coronary artery disease. Data from 19,867 patients from 8 randomized controlled trials were pooled; 1,602 (8.1\%) patients had pre-existing PAD.

The presence of PAD was associated with increased mortality and higher rates of myocardial infarction at 7 days, 30 days and 6 months post-procedure, and with raised mortality at 1 year. Multivariable analyses revealed presence of PAD to be an independent predictor of post-PCI mortality at 30 days (PAD $1.4 \%$ vs no PAD $0.6 \% ; P=0.039), 6$ months $(3.8 \%$ vs $1.4 \% ; P<0.001)$ and 1 year $(4.5 \%$ vs $2.0 \%$; $P=0.013)$. Patients with PAD also tended to have a greater risk of major $(4.8 \%$ vs $3.9 \%$; $P=0.062)$ and minor ( $8.0 \%$ vs $6.8 \% ; P=0.066)$ bleeding events following $\mathrm{PCl}$ than those without PAD. Use of glycoprotein Ilb/llla inhibitors was associated with better short-term outcomes for patients with PAD than was placebo, but seemed to be associated with a higher incidence of major bleeding events.

The authors propose that concomitant PAD increases the systemic atherosclerotic burden on a patient, causing increased mortality after $\mathrm{PCl}$. They suggest that randomized controlled trials focusing on the effects of PAD following $\mathrm{PCl}$ would confirm their work, but state that these are unlikely to be performed.

Original article Saw J et al. (2006) The influence of peripheral arterial disease on outcomes: a pooled analysis of mortality in eight large randomized percutaneous coronary intervention trials. J Am Coll Cardiol 48: 1567-1572

\section{Prevention of vasovagal syncope with physical counterpressure maneuvers}

Physical counterpressure maneuvers (PCMs) should be first-line therapy for patients who suffer recurrent vasovagal syncope with prodromal symptoms, according to a paper by van Dijk et al. There is currently no evidencebased therapy for the condition-treatment consists of explaining the underlying process to patients and dispensing lifestyle advice so that sufferers can recognize triggers and avoid impending episodes.

The PC-Trial initially comprised 223 patients recruited from 15 medical centers worldwide between March 2003 and December 2004. Patients were randomly assigned to receive conventional therapy alone, or in combination with biofeedback-supported PCM training. PCMs included crossing the legs, and tensing the arm or hand muscles. Logbooks were issued so that patients could record symptoms and episodes. Data were checked every 3 months by supervising physicians who were not blinded to group randomization.

After a mean follow-up period of approximately 14 months, final results were available for 208 patients. In the group of patients who received conventional therapy alone, 56 out of $110(50.9 \%)$ experienced recurrent syncope, 